\title{
Cardiometabolic and psychological effects of dual-release hydrocortisone: a cross-over study
}

\author{
Rosemary Dineen', Julie Martin-Grace', Khalid Mohamed Saeed Ahmed², Isolda Frizelle², Anjuli Gunness², \\ Aoife Garrahy', Anne Marie Hannon', Michael W O'Reilly', Diarmuid Smith'1, John McDermott ${ }^{3}$, \\ Marie-Louise Healy4, Amar Agha', Agnieszka Pazderska', James Gibney², \\ Chris J Thompson', Lucy-Ann Behan² and Mark Sherlock' \\ ${ }^{1}$ Academic Department of Endocrinology, Beaumont Hospital/RCSI, ${ }^{2}$ Robert Graves Institute of Endocrinology, \\ Tallaght University Hospital, ${ }^{3}$ Department of Endocrinology, Connolly Hospital, and ${ }^{4}$ Department of Endocrinology, \\ St James Hospital, Dublin, Ireland \\ Correspondence \\ should be addressed \\ to $M$ Sherlock \\ Email \\ marksherlock@beaumont.ie
}

\section{Abstract}

Background: Adrenal insufficiency (Al) is associated with increased cardiovascular morbidity and mortality and reduced quality of life (QoL). Optimum glucocorticoid (GC) dosing and timing are crucial in the treatment of Al, yet the natural circadian secretion of cortisol is difficult to mimic. The once-daily dual-release hydrocortisone (DR-HC) preparation (Plenadren ${ }^{\circledR}$ ), offers a more physiological cortisol profile and may address unmet needs.

Methods: An investigator-initiated, prospective, cross-over study in patients with Al. Following baseline assessment of cardiometabolic risk factors and QoL, patients switched from their usual hydrocortisone regimen to a once-daily dose equivalent of DR-HC and were reassessed after 12 weeks of treatment.

Results: Fifty-one patients (21 PAI/30 SAl) completed the study. Mean age was 41.6 years (s.D. 13), and $58 \%(n=30)$ were male. The median daily HC dose before study entry was $20 \mathrm{mg}$ (IQR 15-20 mg). After 3 months on DR-HC, the mean SBP decreased by $5.7 \mathrm{mmHg}, P=0.0019$ and DBP decreased by $4.5 \mathrm{mmHg}, P=0.0011$. There was also a significant reduction in mean body weight $(-1.23 \mathrm{~kg}, P=0.006)$ and $\mathrm{BMI}\left(-0.3 \mathrm{~kg} / \mathrm{m}^{2}, P=0.003\right)$. In a sub-analysis, there was a greater reduction in SBP observed in patients with SAI when compared to PAI post-DR-HC. Patients reported significant improvements in QoL using three validated QoL questionnaires, with a greater improvement in PAI.

Conclusion: Dual-release hydrocortisone decreases BP, weight and BMI compared with conventional HC treatment, even at physiological GC replacement doses. Additionally, DR-HC confers significant improvements in QoL compared to immediate-release HC, particularly in patients with PAl, which is also reflected in the patient preference for DR-HC.

\section{Introduction}

Adrenal insufficiency (AI) is caused by the primary failure of the adrenal cortex (primary adrenal insufficiency (PAI), most commonly due to autoimmune adrenalitis) or due to disruption of the hypothalamic-pituitary-adrenal axis resulting in loss of stimulation of the adrenal cortex by adrenocorticotropin hormone (ACTH) (secondary adrenal insufficiency (SAI)) (1). All patients with AI receive glucocorticoid (GC) replacement therapy, which aims to replicate physiological cortisol secretion and maintain health. However, despite the availability of GC replacement therapy for several decades, patients with AI have increased morbidity $(2,3,4,5)$, and impairment in quality of life (QoL) compared to the background population (6). Without the availability of reliable biomarkers, conventional 
GC replacement therapy can lead to temporary levels of supraphysiologic cortisol exposure (which in the long term can potentially lead to adverse metabolic effects) (7) or insufficient levels of circulating cortisol which places patients at risk from the consequences of hypoadrenalism, including adrenal crisis and impaired quality of life.

To mimic the physiological rhythm of endogenous cortisol, novel modified-release hydrocortisone preparations have been developed. The dual-release formulation of hydrocortisone (DR-HC), Plenadren ${ }^{\circledR}$, has been licenced for use in clinical practice and is taken once daily in the morning. Plenadren ${ }^{\circledR}$ has been investigated in patients with Addison's disease showing improvements in cardiovascular risk factors, including a reduction in body weight, haemoglobin A1c and blood pressure, as well as a significant improvement in fatigue $(8,9)$. More recently, the DREAM study reported that 24 weeks of DR-HC treatment in patients with AI lead to reduced body weight, normalisation of the immune cell profile, reduced recurrent infections, and improved quality of life compared to patients on conventional glucocorticoid therapy (10).

In the initial phase III study by Johannsson et al. (8) and in the DREAM trial (10), which are the largest prospective trials investigating the DR-HC formulation (Plenadren ${ }^{\circledR}$ ), the majority of study participants were receiving a mean daily dose of hydrocortisone $\geq 25 \mathrm{mg} /$ day $(68.2 \%$ of patients in the phase III trial (8), while the mean daily dose of hydrocortisone in the DREAM trial participants was 26.3 (s.D. 9.4) $\mathrm{mg} /$ day) (11). Therefore, it is debated that the beneficial effects demonstrated in these studies may be explained by a reduction in total daily cortisol exposure from a relatively high dose of hydrocortisone replacement therapy (12). In a prospective study by Behan et al. higher doses of HC produced unphysiological cortisol exposure compared to a matched control group (13).

Several prospective studies investigating DR-HC have included patients who switched from differing glucocorticoid preparations (i.e. cortisone acetate, hydrocortisone, prednisolone or dexamethasone) $(10,14$, 15). In the DREAM study, $57 \%$ of patients were switched from cortisone acetate to DR-HC. A presumed equivalence of $80 \%$ was used for these patients who were switched from cortisone acetate to DR-HC, which is not validated and therefore influences the interpretation of the results. Furthermore, there remains a paucity of data relating to DR-HC in patients with secondary AI, with the majority of studies including retrospective data $(16,17)$, or small study numbers $(14,15,18,19)$.
On this background, our study aimed to assess, in a prospective manner, the effect of changing from immediate-release hydrocortisone to once-daily DR-HC on cardiovascular risk factors and QoL in both primary and secondary AI patients who all switched from the same GC preparation, immediate-release hydrocortisone, and were on physiological GC replacement doses, (median daily HC dose of $20 \mathrm{mg}$ (IQR 15-20 mg)).

\section{Methods}

\section{Study design and population}

We performed an investigator-led, open-labelled, multisite, prospective study at two University Hospitals in Ireland (Tallaght University Hospital and Beaumont Hospital). Eligible patients were male and female patients aged $\geq 18$ years, with a diagnosis of adrenal insufficiency, either PAI or SAI (basal cortisol $<100 \mathrm{nmol} / \mathrm{L}$, peak cortisol $<400$ $\mathrm{nmol} / \mathrm{L}$ after stimulation) confirmed on dynamic testing at diagnosis, who were on conventional hydrocortisone replacement therapy, without any adjustment in hormone replacement for at least 3 months before study entry. Exclusion criteria included; age $<18$ years; pregnancy; patients with acute medical or surgical illness; patients with advanced cardiac/pulmonary disease; patients with a terminal illness; patients on glucocorticoids for purposes other than adrenal insufficiency; patients on agents that interfere with corticosteroid metabolism including oral oestrogen-containing preparations due to the effect on corticosteroid-binding globulin (CBG) and thus total cortisol concentrations; and patients with known Type 1 or Type 2 diabetes mellitus requiring insulin therapy. The clinical characteristics of the patients are summarised in Table 1.

The study was approved by the Joint Research Ethics Committee of Tallaght University Hospital/St James's Hospital and the Beaumont Hospital Research Ethics Committee. Written informed consent was obtained from all patients before participation. All patients had an emergency kit and a steroid emergency card and received education regarding the management of an adrenal crisis.

This study was registered with ClinicalTrials.gov, NCT03282487.

\section{Study intervention}

The investigational medicinal product in this study is the DR-HC, Plenadren ${ }^{\circledR}$ which was prescribed according 
Table 1 Age, gender, duration and aetiology of Al in the study population.

\begin{tabular}{|c|c|}
\hline Characteristics & Values \\
\hline Primary $\mathrm{Al}, n$ & 21 \\
\hline Age (years) mean (S.D.) & $41.05(13.35)$ \\
\hline Male: Female & $9: 12$ \\
\hline Duration of disease (years) mean (S.D.) & $9.8(9.2)$ \\
\hline \multicolumn{2}{|l|}{ Aetiology } \\
\hline \multicolumn{2}{|l|}{ Autoimmune } \\
\hline Isolated & 9 \\
\hline Isolated with coeliac disease & 1 \\
\hline Isolated with vitamin B12 deficiency & 1 \\
\hline APS2 with hypothyroidism & 7 \\
\hline \multicolumn{2}{|l|}{ Bilateral adrenalectomy } \\
\hline VHL- bilateral pheochromocytoma & 1 \\
\hline Carney complex- PPNAD & 1 \\
\hline $\mathrm{BMAH}$ & 1 \\
\hline Secondary Al, $n$ & 30 \\
\hline Age (years) mean (S.D.) & $47.5(12.77)$ \\
\hline Male: Female & $22: 8$ \\
\hline Duration of disease (years) mean (s.D.) & $12.2(7.5)$ \\
\hline \multicolumn{2}{|l|}{ Aetiology } \\
\hline Isolated ACTH & 2 \\
\hline Craniopharyngioma & 5 \\
\hline NFPA & 14 \\
\hline Germinoma & 2 \\
\hline Parasellar meningioma & 1 \\
\hline Apoplexy & 1 \\
\hline Macroprolactinoma & 2 \\
\hline ACTH-dependent Cushing's (in remission) & 2 \\
\hline Congenital hypopituitarism & 1 \\
\hline
\end{tabular}

ACTH, adrenocorticotrophic hormone; Al, adrenal insufficiency; APS, autoimmune polyglandular syndrome; $\mathrm{BMAH}$, bilateral macronodular adrenal hyperplasia; NFPA, non-functioning pituitary adenoma; PPNAD, primary pigmented nodular adrenocortical disease; VHL, Von Hippel Lindau disease.

to the Summary of Product Characteristics (SmPC). The dose of Plenadren ${ }^{\circledR}$ prescribed was equal to the total daily dose of the patients' usual conventional hydrocortisone before study entry. Plenadren ${ }^{\circledR}$ is available in 5 and $20 \mathrm{mg}$ tablets only, hence all patients received their dosage using these formulations. Patients were instructed to take the Plenadren ${ }^{\circledR}$ tablets orally in the fasting state upon waking. In the event of intercurrent illness, recommendations were given to double the daily dose requirement (second dose $8 \mathrm{~h}$ after the first dose) or tripled (6 and $12 \mathrm{~h}$ after the first dose).

After screening for eligibility and informed consent, patients presented for Visit 1. Thereafter, if biochemical investigations were within the normal reference range, patients were switched from immediate-release $\mathrm{HC}$ to the daily dose equivalent of once-daily DR-HC, as per the SmPC, for 12 weeks. At the end of the intervention treatment period, patients presented for Visit 2, thereafter patients were switched back to their usual immediate release $\mathrm{HC}$ regimen, as Plenadren ${ }^{\circledR}$ is currently not available in clinical practice in Ireland, and were followed up in the outpatient clinic according to the standard surveillance protocol of the clinic.

\section{Study procedures}

At baseline and 12 weeks after DR-HC, patients presented after an overnight fast at 08:00 h. Blood pressure was obtained as per NICE guidance, Hypertension in Adults: diagnosis and management (20). Heart rate (b.p.m.), body temperature $\left({ }^{\circ} \mathrm{C}\right)$, respirations (breaths per minute), height $(\mathrm{cm})$, waist and hip circumference $(\mathrm{cm})$ were measured and recorded. Body composition analysers (Tanita ${ }^{\circledR}$ BC418MA for patients recruited in Tallaght University Hospital; and Tanita ${ }^{\circledR}$ DC360S for patients in Beaumont Hospital) were used to calculate BMI, body fat percentage (\%), body fat mass (BFM) (kg), fat-free mass (FFM) (kg), muscle mass $(\mathrm{kg})$, total body water percentage, and basal metabolic rate.

Fasting laboratory investigations were performed including routine renal/bone/liver profiles (sodium, potassium, urea, creatinine, calcium, alkaline phosphatase (ALP), magnesium, alanine aminotransferase (ALT), gamma-glutamyl transferase (GGT), bilirubin) serum albumin, fasting total cholesterol, HDL- and LDLcholesterol, triglycerides, haemoglobin A1c, C-reactive protein and full blood count using in-hospital assays.

All study participants completed three validated QoL questionnaires; the Short Form-36 Health Survey (SF-36) (21), the Addison's Disease-specific, AddiQoL questionnaire (22), and the Nottingham Health Profile (NHP) (23). The SF-36 aims to assess general well-being over the preceding 4 weeks by evaluating responses to statements through 8 domains of health. Scores are coded and transformed to a scale of 0-100 with higher scores indicating a better QoL (21). The AddiQoL questionnaire has been validated for patients with PAI is a 30-item questionnaire (22). Positive items have scores from 1 to 6; negative QoL statements were reversed for questionnaire scoring (6 to 1$)$. The scoring was converted to points: $6=4$ points; 5 and $4=3$ points; 3 and $2=2$ points, $1=1$ point. The algebraic sum of points was calculated. Higher points indicate a better QoL. The NHP contains 38 yes/ no questions over 6 health domains. Each question is weighted and the sum of each domain is $0-100$ with higher scores indicating worse QoL (23).

To strengthen the QoL assessment, we recruited healthy age- and BMI-matched controls from similar socio-economic background to our patient population, to complete the same QoL questionnaires. 


\section{Outcomes}

The primary efficacy outcome was bodyweight changed from baseline to 12 weeks.

Assuming a s.D. of $1.3 \mathrm{~kg}$ for bodyweight change, based on published data (8), and $1 \mathrm{~kg}$ as the minimal clinically relevant difference between treatments, we estimated that a sample size of 54 would be needed to provide $80 \%$ power to detect a $1.0 \mathrm{~kg}$ difference in the primary outcome, with a two-sided significance level of 0.05 , assuming that $20 \%$ of patients would drop out of the study. Change from baseline was calculated as the value at 12 weeks minus the baseline value.

Secondary outcomes included a change from baseline to 12 weeks in metabolic profile (assessed by measurement of blood pressure, HbA1c, fasting serum lipids, BMI, waist circumference) and quality of life. Safety was monitored throughout the study and included the recording of adverse events, adrenal crises and symptoms suggestive of hypoadrenalism. An adrenal crisis was defined as per guidelines (24).

\section{Statistical analysis}

The normality of quantitative variables was tested with the Shapiro-Wilk test. The baseline characteristics of the groups are presented as mean (s.D.) or median (interquartile range) as appropriate. The differences between the posttreatment and baseline data were evaluated with paired $t$-tests in a single group for quantitative variables and chisquare for categorical variables or the appropriate nonparametric test. To assess whether the effect in SAI differs from PAI, a formal interaction test was performed.

QoL questionnaires were coded for analysis by the investigators, and the investigators were blind to the study to reduce the risk of bias during the analysis of the results. Investigators were blinded to the study ID and study visit matching each questionnaire. Analysis of QoL involved calculating age and gender-specific mean and S.D. score values from the normally distributed healthy control data to produce age and gender-specific Z-scores for each patient before and after DR-HC. The Z-score reveals how many units of the s.D. each subject is above or below the mean. The Z-score was calculated as follows: $\mathrm{Z}=(\mathrm{x}-\mu) / \mathrm{o}$, where $\mathrm{x}=$ individual $\mathrm{QoL}$ value, $\mu=$ mean QoL value of controls of equal gender and age and $\alpha=$ S.D. of QoL value of controls of equal gender and age. The Z-score was calculated for each domain in SF36, NHP and AddiQoL. A higher score is associated with worse QoL in the NHP, therefore, a positive Z score denotes worse QoL compared to healthy controls. In the SF36 and AddiQoL, a higher score indicates better QoL and therefore a negative Z score denotes decreased QoL compared to healthy controls. Significance was defined for $P$-values less than 0.05. Statistical analysis was performed using GraphPad Prism version 8.2.0 for Windows, GraphPad Software.

\section{Results}

\section{Baseline demographic and metabolic parameters}

From September 2017 to March 2019, 150 patients were screened for eligibility. A consort diagram of the study population is outlined in Fig. 1. Fifty-eight patients were entered into the study. Two patients completed Visit 1 but did not commence the study drug. A further 5 patients withdrew from the study ( 4 had an adverse event and 1 declined to attend for follow-up) therefore, a total of 51 patients completed the study (i.e. with available data from both Visit 1 and Visit 2); 21 patients with PAI and 30 patients with SAI. The mean age of patients with PAI and SAI was similar (Table 1). There was a predominance of female patients in the PAI group and a predominance of men in the SAI. Disease duration and aetiology of AI in the study population are outlined in Table 1.

At study entry, patients with SAI had a significantly higher mean (s.D.) weight (94.8 kg (25.1) vs $76.4 \mathrm{~kg}$ (18.2), $P=0.006)$, BMI $\left(31.5 \mathrm{~kg} / \mathrm{m}^{2}(7.3) \mathrm{vs} 26.8 \mathrm{~kg} / \mathrm{m}^{2}\right.$ (6.7), $P=0.0023)$ and waist circumference $(106.4 \mathrm{~cm}$ (19) vs $88.5 \mathrm{~cm}(14), P=0.0009)$ compared to patients with PAI, however, there were more men in the SAI group which may account for the observed differences (Table 2). Comparison of blood pressure and lipid characteristics of patients with PAI and SAI are presented in Table 2. Both PAI and SAI groups had similar mean blood pressure at baseline, however, a higher proportion of SAI patients were receiving anti-hypertensive therapy $(P=0.03)$. A higher proportion of SAI patients were receiving statin therapy $(P=0.015)$. Patients with PAI were on a higher median daily dose of hydrocortisone replacement compared to SAI patients (20 mg (IQR: 20-25 mg) vs $17.5 \mathrm{mg}$ (IQR: 15-20 $\mathrm{mg}), P=0.0034)$, and were more likely to receive thrice daily hydrocortisone than patients with SAI (Table 2).

\section{Post 12 weeks of dual-release hydrocortisone therapy}

Effect on blood pressure

After 12 weeks of DR-HC treatment, we observed a significant reduction in systolic blood pressure $(-5.7 \mathrm{mmHg}, P=0.0019)$ 


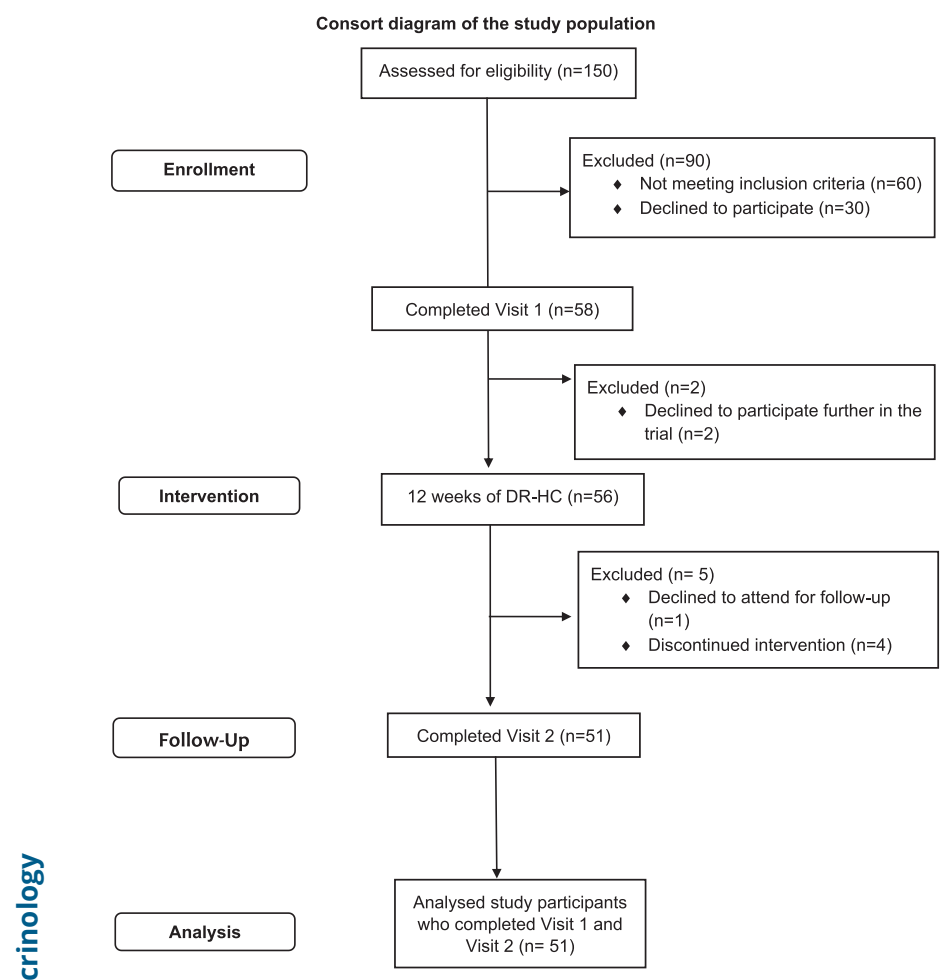

Figure 1

Consort diagram of the study population.

(Fig. 2A) and diastolic blood pressure $(-4.5 \mathrm{mmHg}, P=0.0011)$ (Fig. 2B) within the entire study population $(n=51)$. These observed reductions in SBP and DBP remained significant when adjusted for anti-hypertensive therapy (SBP adjusted $P=0.009$, DBP adjusted $P=0.005$ ). When comparing blood pressure reduction between patients with PAI and SAI, we observed a significant reduction in SBP and DBP in patients with SAI compared to patients with PAI (Fig. 2C, D, E and F).

\section{Effects on body weight, BMI, body composition and waist circumference}

We observed a significant reduction in mean body weight $(-1.23 \mathrm{~kg}, P=0.006)$, within the entire study population $(n=51)$ after 12 weeks of DR-HC (Fig. 3A). This translated to a significant reduction in BMI $(-0.3 \mathrm{~kg} /$ $\mathrm{m}^{2}, P=0.003$ ) (Fig. 3B). There was a reduction in overall waist circumference (WCM) among the study group ( -1 $\mathrm{cm}, P=0.07$ ) but this was not significant (Fig. 3C). Figure 3 also illustrates the observed reductions in body weight, BMI and WCM among the disease subgroups, PAI and SAI. Following 12 weeks of DR-HC, we observed a reduction in mean body weight in patients with SAI ( $-1.48 \mathrm{~kg}$ (s.D. 3.6), $P=0.03)$, compared to patients with PAI ( $-0.9 \mathrm{~kg}$ (s.D. 2.2$)$,
Table 2 Patients with PAI $(n=21)$ and SAI $(n=30)$ at baseline. Data expressed as mean (S.D.) unless otherwise stated.

\begin{tabular}{|c|c|c|c|}
\hline & $\begin{array}{c}\text { Primary Al } \\
(n=21)\end{array}$ & $\begin{array}{c}\text { Secondary } \\
\text { Al }(n=30)\end{array}$ & P value \\
\hline Age (years) & $41.1(13.4)$ & $47.5(12.8)$ & 0.08 \\
\hline Weight (kg) & $76.4(18.2)$ & $94.8(25.1)$ & 0.006 \\
\hline BMI (kg/m²) & $26.8(6.7)$ & $31.5(7.3)$ & 0.002 \\
\hline WCM (cm) & $88.5(14)$ & 106.4 (19) & 0.0009 \\
\hline $\mathrm{SBP}(\mathrm{mmHg})$ & $125.6(15.7)$ & $125.3(14.7)$ & 0.93 \\
\hline $\mathrm{DBP}(\mathrm{mmHg})$ & 75.7 (9) & $78.4(10)$ & 0.34 \\
\hline HR (b.p.m.) & $71.7(11.3)$ & $71.8(9.5)$ & 0.99 \\
\hline Anti-hypertensive therapy $(n)$ & 0 & 6 & 0.03 \\
\hline Statin therapy $(n)$ & 1 & 10 & 0.015 \\
\hline Total Cholesterol (mmol/L) & $4.88(0.97)$ & $4.9(1.01)$ & 0.83 \\
\hline LDL cholesterol (mmol/L) & $2.73(0.7)$ & $2.95(0.94)$ & 0.38 \\
\hline Triglycerides (mmol/L) & $0.79(0.26)$ & $1.1(0.63)$ & 0.05 \\
\hline HDL cholesterol (mmol/L) & $1.79(0.47)$ & $1.48(0.47)$ & 0.02 \\
\hline $\begin{array}{l}\text { HC replacement dose (mg/day) } \\
\text { Median (IQR) } \\
\text { Replacement regimen }\end{array}$ & $20(20-25)$ & $17.5(15-20)$ & 0.003 \\
\hline BID & 12 & 30 & $<0.0001$ \\
\hline TID & 9 & 0 & $<0.0001$ \\
\hline
\end{tabular}

Significance, $P<0.05$.

$\mathrm{BID}$, twice daily; DBP, diastolic blood pressure; HC, hydrocortisone; HR, heart rate; LDL, low-density lipoprotein; PAl, primary adrenal insufficiency; SAI, secondary adrenal insufficiency; SBP, systolic blood pressure; TID, thrice daily.

$P=0.07$ ) (Fig. 3A), however, patients with PAI did have a significant reduction in mean BMI $\left(-0.34 \mathrm{~kg} / \mathrm{m}^{2}, P=0.04\right)$ (Fig. 3B). Patients with SAI also had a significant mean reduction in waist circumference $(-2.7 \mathrm{~cm}, P=0.014)$ (Fig. 3C). We did not find any significant differences in bioimpedance analysis post-DR-HC therapy among the study population (Supplementary Table 1, see section on supplementary materials given at the end of this article). However, we did observe a positive correlation between the reduction in body weight and the reduction in fat mass $(\mathrm{kg})$ in the study population post-DR-HC $(\mathrm{r}=0.67(95 \%$ CI 0.31-0.75), $P<0.0001$ ) and a trend towards a positive correlation between the reduction in body weight and the reduction in SBP observed in the patient population, but this was not statistically significant $(\mathrm{r}=0.27$ (95\% CI $0.02-0.5), P=0.06$ ).

\section{Effect on fasting lipids, HbA1c and haematology parameters}

No differences in fasting cholesterol or HbA1c were observed after the 12 weeks of DR-HC. We observed a small but significant increase in mean fasting triglycerides after 12 weeks of DR-HC therapy $(+0.1 \mathrm{mmol} / \mathrm{L}(0.02)$, $P=0.03$ ). Due to a complementary in-vivo component of our study (where the patient received an overnight tablet of dexamethasone) fasting glucose and insulin could not be interpreted accurately. 


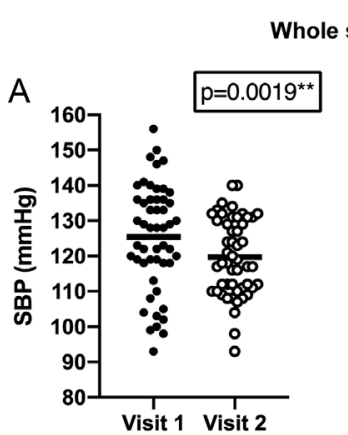

Whole study cohort $(n=51)$
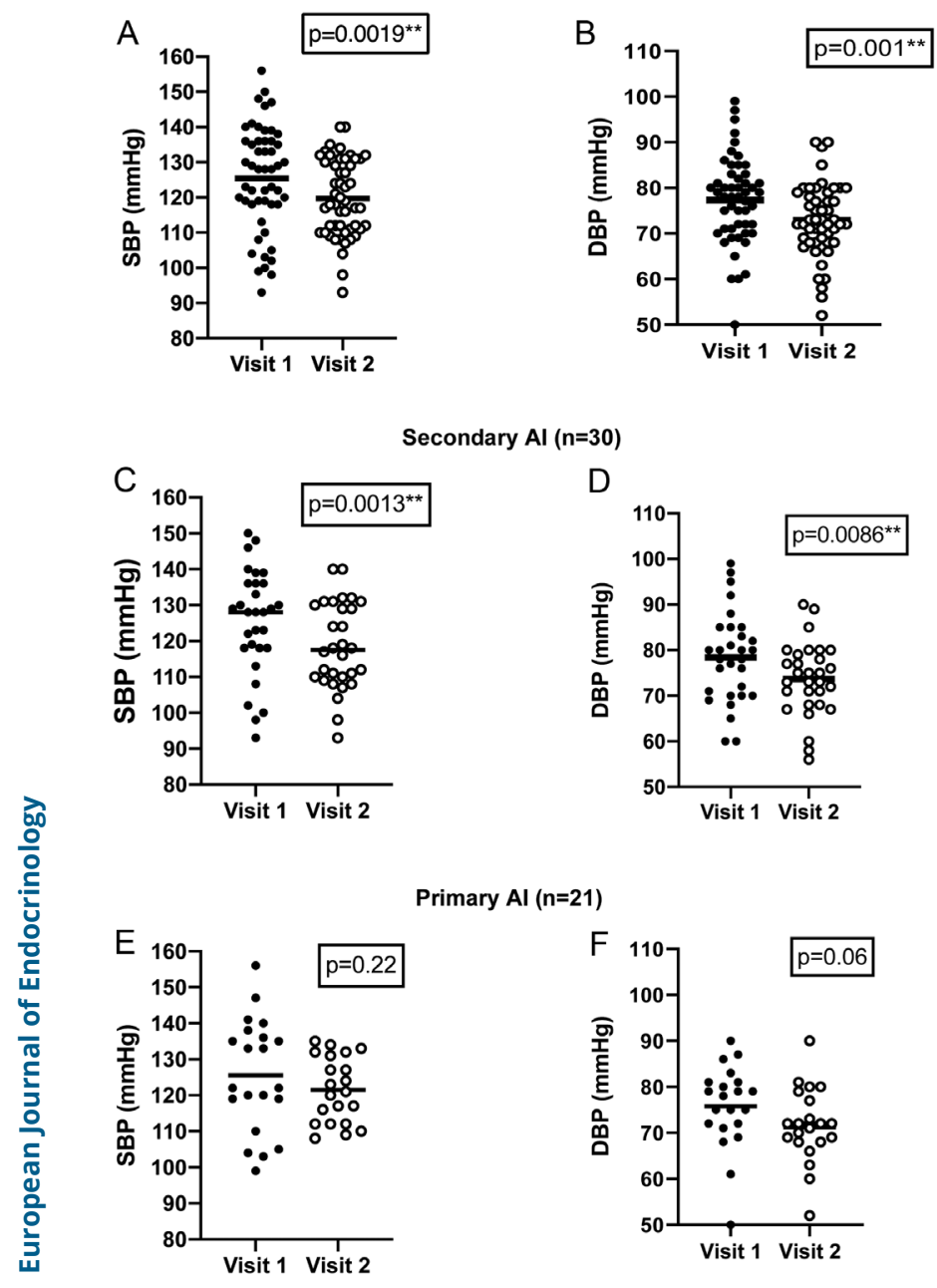

Primary Al (n=21)

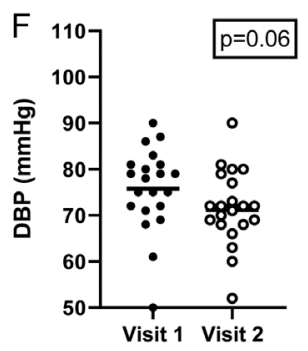

Figure 2

Blood pressure on conventional immediate release $\mathrm{HC}$ (visit 1) and 12 weeks after dual-release hydrocortisone (visit 2). (A) SBP pre and post 12 weeks of DR-HC in the entire study population. (B) DBP pre and post 12 weeks of $\mathrm{DR}-\mathrm{HC}$ in the entire study population. (C) SBP pre and post 12 weeks of DR-HC in patients with PAI. (D) DBP pre and post 12 weeks of DR-HC in patients with PAI. (E) SBP pre and post 12 weeks of DR-HC in patients with SAI. (F) DBP pre and post 12 weeks of DR-HC in patients with SAI. SBP, systolic blood pressure; DBP, diastolic blood pressure; DR-HC, dual-release hydrocortisone; PAI, primary adrenal insufficiency; SAl, secondary adrenal insufficiency.

There was a significant reduction in the mean total white cell count (WCC) between baseline and post 12 weeks of DR-HC $\left(7.03 \times 10^{3} / \mu \mathrm{L}\right.$ vs $6.56 \times 10^{3} / \mu \mathrm{L}$, $P=0.005)$, with a significant reduction in neutrophil count $\left(5.06 \times 10^{3} / \mu \mathrm{L}\right.$ vs $\left.4.63 \times 10^{3} / \mu \mathrm{L}, P=0.002\right)$, but not in lymphocyte count.
We observed a small but significant reduction in the mean concentration of the liver enzyme, alanine aminotransferase $(-2.5 \mathrm{mmol} / \mathrm{L}, P=0.04)$ following 12 weeks of DR-HC. No statistical changes occurred in the concentration of bilirubin, alkaline phosphatase and gamma-glutamyl transpeptidase post 12 weeks of DR-HC therapy. Similarly, no statistically or clinically relevant changes were observed in other haematology parameters such as haemoglobin, haematocrit, platelet count, and in routine biochemistry including electrolytes, and bone profile.

\section{Effect on quality of life (QoL)}

In the SF-36, there was a significant improvement reported among the study population after 12 weeks of DR-HC in the physical and emotional role domains, in addition to a significant improvement in the domains of vitality, and mental health (Table 3). The AddiQoL was also assessed among the entire study cohort before and after DR-HC, and there was a significant improvement in mean QoL scores across all domains including; fatigue, symptoms, emotions and miscellaneous which includes sleep, sexuality and impact of intercurrent illness (Table 3). In the NHP, lower scores indicate better QoL. Patients reported significant improvement in energy levels after 12 weeks of DR-HC therapy (Table 3).

Using calculated Z scores (the s.D. from the mean of the normative population), we observed the same significant differences between Visit 1 (when patients were on conventional hydrocortisone) and Visit 2 (after 12 weeks of DR-HC), as outlined previously, which are illustrated in Fig. 4. In the SF-36 there was a reduction in the negative $Z$ score in the physical role $(P=0.001)$, emotional $(P=0.02)$ role, vitality $(P=0.0014)$, and mental health $(P=0.03)$, after DR-HC compared to controls, signifying an improvement. Similarly, there was a reduction in the negative $\mathrm{Z}$ score in all domains of the AddiQoL after DR-HC, reflecting an improvement in QoL. In the NHP, we observed only a significant improvement post-DR-HC in the energy level domain (highlighted by a decrease in the positive $Z$ value).

When examining results by disease group, we observed greater improvement in QoL post-DR-HC in patients with PAI compared to patients with SAI (Fig. 5). Patients with PAI reported significant improvement in all domains of the AddiQoL (Fig. 5D), in addition to an improvement in the 


$$
\text { A }
$$
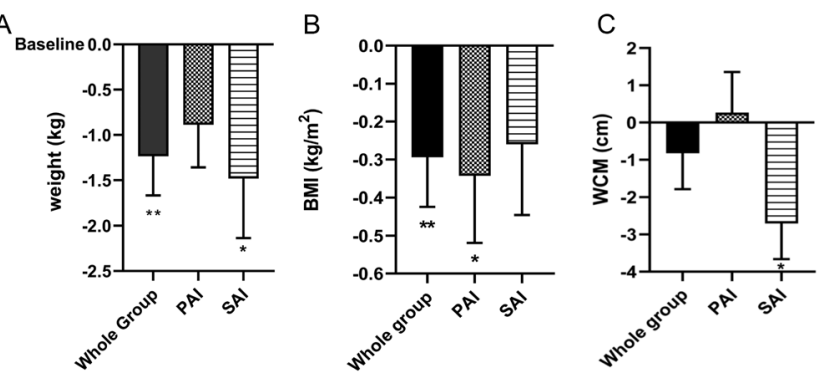

\section{Figure 3}

Change in (A) weight, (B) BMI and (C) waist circumference (WCM) in the study patients from baseline to 12 weeks post-dual-release hydrocortisone. Whole group $=51$ patients; Primary adrenal insufficiency $(\mathrm{PAI})=21$ patients; Secondary adrenal insufficiency (SAI) $=30$ patients. Data expressed as mean (S.E.M.) unless otherwise stated, $* P$ value $<0.05, * * P$ value $<0.01$. WCM, waist circumference; PAl, primary adrenal insufficiency; SAl, secondary adrenal insufficiency.

physical role domain $(P=0.005)$, vitality $(P=0.003)$, and mental health $(P=0.04)$ in the SF-36 (Fig. 5A). For patients with SAI, all mean QoL scores improved postDR-HC but only the domains of bodily pain $(P=0.04)$ and the emotional role $(P=0.016)$ in the SF-36 were significant (Fig. 5D).

Patient preference for either once daily DR-HC or conventional therapy was assessed at the end of the study, and $100 \%$ of patients chose to continue DR-HC if given the option.

\section{Adverse events}

No deaths occurred during the study. Three patients withdrew from the trial after reporting adverse events including fatigue and nausea, all occurring within 2 weeks of commencing the treatment. Two patients were hospitalised and treated with intravenous steroids with acute adrenal insufficiency triggered by infectious disorders (one case of bacterial gastroenteritis and one case of pneumonia). These two cases were deemed serious due to hospitalisation and occurred at least 4 weeks after DR-HC commencement. The patient with gastroenteritis continued DR-HC for the remainder of the study without adverse effects, while the patient with pneumonia discontinued the intervention.

The percentage of days when increased hydrocortisone use reported during the 3 months of DR-HC therapy was low (5\% of study days), similar to that reported in a recent study by Schöfl et al. (25).

\section{Discussion}

Our prospective study shows that in patients with adrenal insufficiency (both PAI and SAI), the switch from conventional HC replacement therapy, at physiological replacement doses, to once-daily DR-HC is associated with significant improvements in blood pressure, body weight, BMI and QoL.

Similar to previous studies $(9,10,14)$, we observed a significant reduction in weight and BMI in the entire study cohort, with an additional reduction in waist circumference observed in patients with SAI. This reduction in body weight may be related to the reduction in the 24-h exposure and supraphysiological peaks of circulating cortisol with DR-HC compared to immediaterelease HC, as shown in previous pharmacokinetic studies $(8,26,27)$. In the study by Johannsson and colleagues (8), the reduced area under the curve (AUC) of circulating cortisol obtained with DR-HC compared with immediaterelease $\mathrm{HC}$ regimen was predominantly due to lower cortisol concentrations in the afternoon and evening. Studies have shown that treatment with immediaterelease HC is associated with supraphysiological cortisol exposure in the late afternoon and evening (13). DR-HC displays a similar cortisol time-exposure profile to the endogenous cortisol profile, avoiding the fluctuations in cortisol concentration that occur with conventional hydrocortisone therapy (8).

We observed a mean reduction in SBP of $-5.7 \mathrm{mmHg}$ and DBP of $-4.5 \mathrm{mmHg}$ post 12 weeks of DR-HC. Our study population were receiving a lower median daily HC dose of $20 \mathrm{mg}$ at study entry compared to previous studies reporting a similar BP reduction (8), demonstrating that the beneficial effect on BP is not only observed at higher mean daily HC doses, as previously debated (12). Importantly, earlier studies that have focused on simply a reduction in the daily cumulative $\mathrm{HC}$ dose using immediate-release hydrocortisone failed to demonstrate any benefit on BP $(28,29,30)$. We observed a greater reduction in BP in patients with SAI compared to patients with PAI postDR-HC, which has not been previously reported. Some patients with SAI may have partial ACTH deficiency, with higher circulating endogenous cortisol levels than patients with PAI. These patients may, therefore, be overreplaced on similar doses of HC replacement to patients with PAI. However, the patients in our study had evidence of severe ACTH deficiency confirmed on dynamic testing at diagnosis and were on a median daily HC dose of 17.5 $\mathrm{mg}$ (IQR 15-20 mg), therefore were unlikely to be overreplaced before initiation of DR-HC. 
Table 3 Absolute quality of life scores in patients with adrenal insufficiency at baseline, on conventional immediate release hydrocortisone (Visit 1), after 12 weeks of dual-release hydrocortisone (Visit 2), and age and BMI-matched healthy control population from a similar socio-economic background. Data expressed asmean (S.D.)

\begin{tabular}{|c|c|c|c|c|c|c|c|}
\hline & \multicolumn{3}{|c|}{$\begin{array}{l}\text { QoL in patients with Al on conventional } \\
\text { HC compared to healthy controls }\end{array}$} & \multicolumn{3}{|c|}{$\begin{array}{l}\text { QoL in patients with AI before (Visit 1) } \\
\text { and after } 12 \text { weeks of DR-HC (Visit 2) }\end{array}$} & \multirow{2}{*}{$\begin{array}{c}\text { Effect size } \\
\text { Cohens } \boldsymbol{d}(95 \% \mathrm{Cl})\end{array}$} \\
\hline & (Visit 1§) & Controls & $P$ value & Visit 1 & Visit 2 & $P$ value & \\
\hline \multicolumn{8}{|c|}{ Short Form-36 (High scores indicate better QoL)* } \\
\hline Physical functioning & $79.8(19.3)$ & $98.6(2.9)$ & 0.0001 & $79.8(19.3)$ & $80.8(20.31)$ & 0.69 & $0.05(-0.34,0.44)$ \\
\hline Role physical & $60(39.5)$ & $100(0)$ & $<0.0001$ & $60(39.5)$ & $76(36.1)$ & 0.003 & $0.42(0.03,0.81)$ \\
\hline Bodily pain & $76(24.8)$ & $85.4(13)$ & 0.13 & $76(24.8)$ & $72.6(27.5)$ & 0.23 & $-0.13(-0.76,0.51)$ \\
\hline General health & $55.6(20.9)$ & $88.2(11.8)$ & $<0.0001$ & $55.6(20.9)$ & $57.2(21.9)$ & 0.39 & $0.07(-0.56,0.71)$ \\
\hline Vitality & $34.2(17)$ & $57.3(9.7)$ & $<0.0001$ & $34.2(17)$ & $41.6(19.9)$ & 0.001 & $0.4(-0.25,1.03)$ \\
\hline Social functioning & $75.8(24.4)$ & $96.5(8.4)$ & 0.0008 & $75.8(24.4)$ & $80(23.6)$ & 0.16 & $0.17(-0.47,0.81)$ \\
\hline Role emotional & $70(37.65)$ & $92.6(18.3)$ & 0.018 & $70(37.7)$ & $81.3(31.7)$ & 0.048 & $0.32(-0.32,0.96)$ \\
\hline Mental health & $73.8(17.34)$ & $87.3(9.9)$ & 0.003 & $73.8(17.3)$ & $77.2(19.3)$ & 0.032 & $0.19(-0.46,0.82)$ \\
\hline \multicolumn{8}{|c|}{ Nottingham Health Profile (Low scores indicate better QoL) } \\
\hline Energy level & $36.3(38.9)$ & $2.2(9.2)$ & $<0.001$ & $36.3(38.9)$ & $25.3(36.2)$ & 0.028 & $0.29(-0.35,0.93)$ \\
\hline Pain & $13.0(24.6)$ & $1.1(4.8)$ & 0.04 & $13.0(24.6)$ & $13.4(26.7)$ & 0.49 & $0(-0.64,0.633)$ \\
\hline Emotional reaction & $15.9(22)$ & $4.2(15.3)$ & 0.04 & $15.9(22)$ & $12.3(20.6)$ & 0.059 & $0.17(-0.47,0.8)$ \\
\hline Sleep & $19.3(25)$ & $6.6(18.5)$ & 0.03 & $19.3(25)$ & $17.3(25.7)$ & 0.38 & $0.08(-0.56,0.71)$ \\
\hline Social isolation & $11.2(24.5)$ & $0(0)$ & 0.06 & $11.2(24.5)$ & $13.1(27.7)$ & 0.31 & $0.07(-0.71,0.56)$ \\
\hline Physical abilities & $8.2(13.2)$ & $0.2(3.5)$ & 0.03 & $8.2(13.2)$ & $8.0(13.4)$ & 0.67 & $0.01(-0.62,0.65)$ \\
\hline \multicolumn{8}{|c|}{ AddiQol (High scores indicate better QoL) } \\
\hline Fatigue & $30.9(8.3)$ & $43.2(3)$ & $<0.0001$ & $30.9(8.3)$ & $34.1(7.8)$ & 0.001 & $0.37(-0.28,1.01)$ \\
\hline Symptoms & $40.5(10)$ & $50.8(2.4)$ & $<0.0001$ & $40.5(10)$ & $43.7(7.7)$ & 0.0006 & $0.35(-0.29,0.99)$ \\
\hline Emotions & $35(6.9)$ & 42.4 (3.9) & $<0.0001$ & $35(6.9)$ & $37.0(6.7)$ & 0.016 & $0.29(-0.35,0.93)$ \\
\hline $\begin{array}{l}\text { Miscellaneous (sleep, sexuality, } \\
\text { and impact of intercurrent } \\
\text { disease, six items) }\end{array}$ & $18.2(5.0)$ & $25.5(2.5)$ & $<0.0001$ & $18.2(5.0)$ & $19.9(5.1)$ & $0.019 *$ & $0.33(-0.31,0.97)$ \\
\hline
\end{tabular}

Significance $P<0.05$. *Marked out of 100 ; ${ }^{5}$ Represents patients with Al at baseline.

$\mathrm{Al}$, adrenal insufficiency; DR-HC, dual-release hydrocortisone.

All-cause mortality is increased in patients with hypopituitarism when compared with age- and sexmatched controls (31), and this is predominantly due to cardiovascular disease (CVD) $(32,33,34)$. Cardiovascular risk factors are highly prevalent in adult patients with hypopituitarism (35). A recent systematic review and meta-analysis of all available large-scale blood pressurelowering randomised trials (36), revealed that a $10 \mathrm{mmHg}$ reduction in SBP reduced the risk of cardiovascular disease events by $20 \%$, coronary heart disease by $17 \%$, stroke by $27 \%$, heart failure by $28 \%$ and all-cause mortality by $13 \%$. Additionally, they observed that proportional reductions were seen in trials that included patients with lower baseline systolic blood pressure $(<130 \mathrm{mmHg})$ and major cardiovascular events were reduced in high-risk patients with baseline comorbidities (36). Therefore, a mean SBP reduction of $6.9 \mathrm{mmHg}$ in our patients with SAI (even with baseline SBP $<130 \mathrm{mmhg}$ ) is significant and may translate to a risk reduction in cardiovascular events.

In comparison to the study by Johannsson et al. (8), we did not observe a significant reduction in BP in patients with PAI. However, our study included a smaller number of patients with PAI and therefore was potentially underpowered to appreciate a significant difference. Also, our patients with PAI were receiving a lower median daily HC dose of $20 \mathrm{mg}$ (s.D. 15-20 mg) at study entry, compared to the study by Johannsson et al., where approx. $88 \%$ of patients were receiving a daily $\mathrm{HC}$ dose of $\geq 25 \mathrm{mg}$, with $19 \%$ receiving $40 \mathrm{mg}$ of $\mathrm{HC}$ daily. In patients who receive higher doses of hydrocortisone, there is a potential for cortisol to inappropriately activate the mineralocorticoid receptor (MR) (37). From a clinical perspective, a nonstatistical reduction in BP in patients with PAI may be a reassuring observation. Patients with PAI have concurrent mineralocorticoid deficiency, making them vulnerable to fluctuations in salt and water balance and therefore blood pressure homeostasis.

We observed a significant reduction in the mean total WBC count (and in particular a reduction in neutrophil count) post 12 weeks of DR-HC. This is a potential further beneficial effect of DR-HC as several epidemiological studies have shown a positive correlation between WBC count and risk of cardiovascular disease both in healthy 
A

SF-36 Z scores

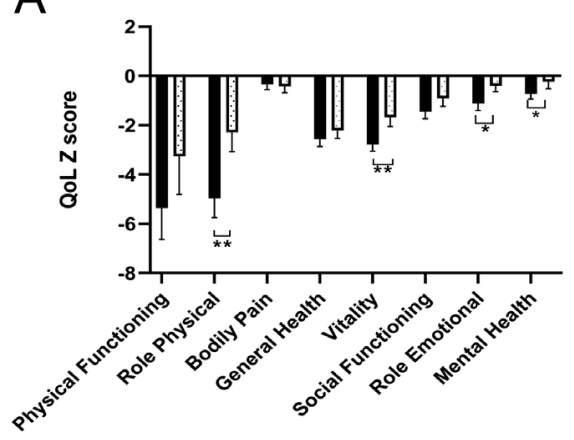

B

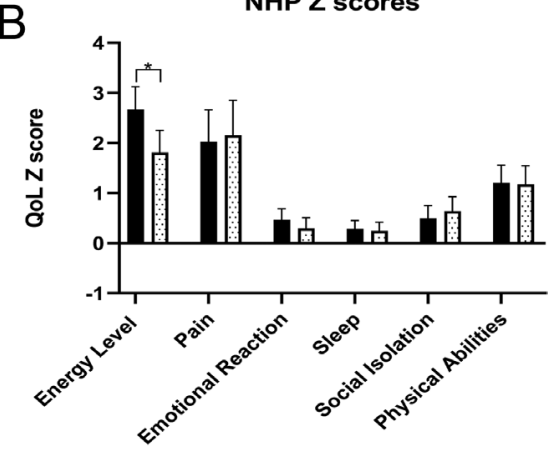

C

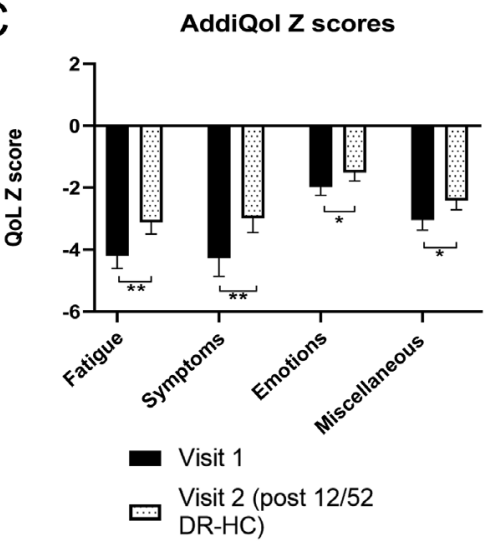

Figure 4

Quality of life standard deviation scores (Z scores) calculated using age- and gender-matched control data for the SF36 (A), NHP (B) and AddiQoL (C) questionnaires between Visit 1 (on conventional hydrocortisone) and Visit 2 (post 12 weeks of dual-release hydrocortisone) in the entire cohort. QoL - the quality of life, SF36 - short form 36 QoL questionnaire, NHP Nottingham health profile questionnaire, Z scores - standard deviation scores calculated using age- and gender-matched control data. Data expressed in mean (s.E.m.) unless otherwise stated. $P$ value $>0.05, * P$ value $<0.05, * * P$ value $<0.01, * \star * P$ value $<0.001$. NHP: positive $Z$ score denotes worse QoL compared to healthy controls. SF36 and AddiQoL: negative Z score denotes worse QoL compared to healthy control. subjects and in patients with established CVD (38, 39, $40,41)$. Studies have shown that the higher risk for CVD associated with increased total WBC count seemed to be accounted for by the increased neutrophil count $(42,43)$.

We also observed a small but significant reduction in the mean concentration of the liver enzyme, ALT. Serum ALT has been shown in cross-sectional studies to be associated with the presence of fatty liver on ultrasonography and with liver fat content as measured by MRI spectroscopy (44). Additionally, serum ALT has been proposed as a biomarker for non-alcoholic fatty liver disease (NAFLD) (45). Recent data by Guarnotta who showed an improvement in the hepatic steatosis index post 12 months of DR-HC treatment, however, this study focused on patients with secondary AI only (17). There is a paucity of data investigating NAFLD in patients with primary adrenal insufficiency hence the observed reduction post-DR-HC in our study population, including both PAI and SAI, is interesting and requires further investigation.

We have confirmed, with a more comprehensive set of assessments, the previously reported improvement in QoL associated with DR-HC therapy $(8,10,14,15,46)$. Our study group reported significant improvements in energy, physical roles, emotional roles, vitality and mental health. Patients with PAI reported significant improvements in all domains of the AddiQoL. These self-reported improvements in QoL challenge the argument that the observed reductions in BP, body weight, and BMI may be explained by simply reducing total daily cortisol exposure and potentially rendering the patient hypo adrenal, as numerous studies have shown that insufficient glucocorticoid replacement results in a reported deterioration in QoL (47).

Inadequate GC replacement therapy is suspected to be a major cause of reduced QoL in AI, although evidence has been lacking $(48,49)$. Immediate release $\mathrm{HC}$ given in twice/thrice daily doses is associated with considerable variability in cortisol concentrations, with a substantial proportion of patients being undertreated and overtreated during the same 24-h period (13). DR-HC avoids these multiple daily peaks and troughs in cortisol levels, which may translate to the observed improvement in QoL for these patients.

Transition to once-daily, dual-release hydrocortisone appeared to be safe, with no significant adverse events. Minor adverse events were recorded and perhaps, given the non-blinded nature of the study, patients may have increased awareness of associated signs and symptoms than to a real change in cortisol exposure. We did observe an increase in fasting triglycerides, which has been seen 
A

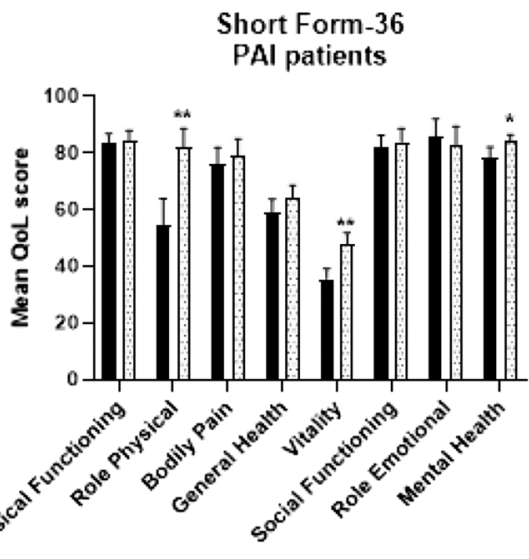

D

\section{Short Form-36}
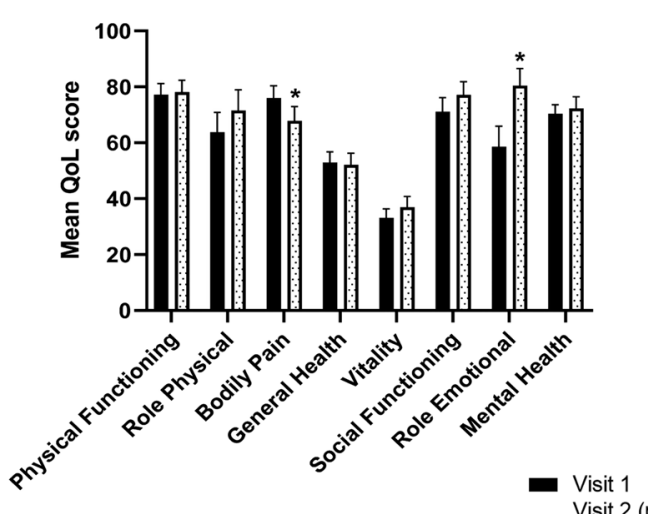

Visit 2 (pos
DR-HC)
B

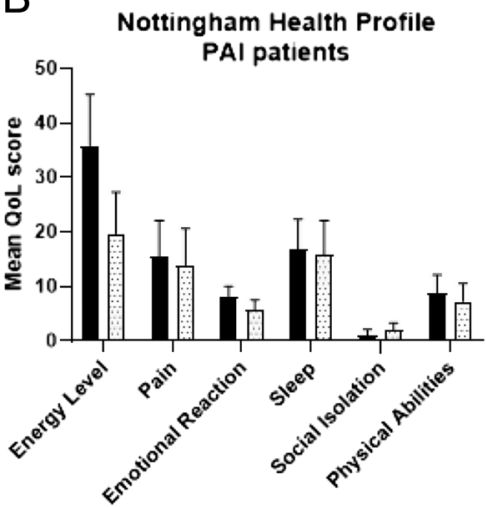

E
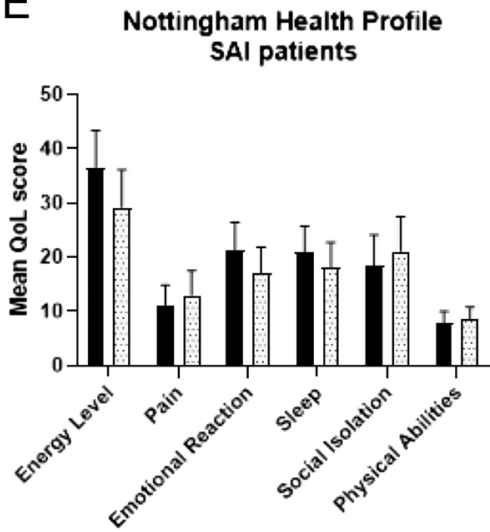

C

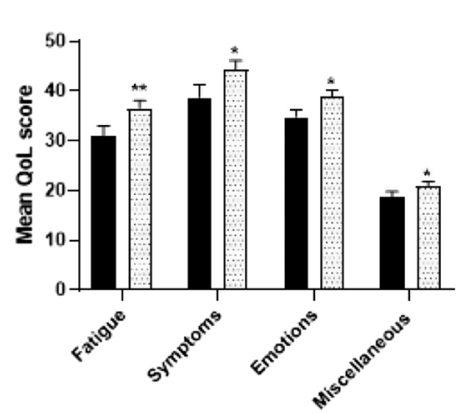

$\mathrm{F}$

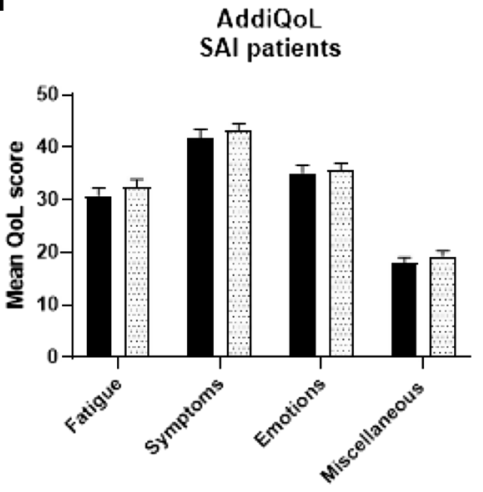

Figure 5

Quality of life scores in patients with PAI $(n=21)$ and SAI $(n=30)$ at baseline, on conventional immediate release hydrocortisone (Visit 1) and post 12 weeks of dual-release hydrocortisone (Visit 2). Data expressed in mean (S.E.M.) unless otherwise stated, significance $P$ value $<0.05$. PAl, primary adrenal insufficiency; SAl, secondary adrenal insufficiency; NHP, Nottingham Health Profile; SF-36, Short Form 36; AddiQoL, Addison's Disease-specific Quality of Life questionnaire. SF-36 and AddiQoL: High scores indicate better QoL, NHP: Low scores indicate better QoL for NHP.

in the previous trials $(8,15)$, and which merits further investigation.

Our study has some limitations. First, patients were not blinded to the treatment (for safety purposes) which could influence subjective parameters such as the QoL questionnaires. Secondly, patients who took part in the study may have been a more motivated patient cohort as they agreed to take part in the study. However, we feel our paper also has strengths including the inclusion of patients with primary and secondary adrenal insufficiency, which allowed comparison of the effect of DR-HC. The relatively lower dose of hydrocortisone used in our cohort compared to previous studies. All our patients were receiving hydrocortisone before a switch to DR-HC in comparison to previous studies which have had patients who have switched from other glucocorticoids (such as prednisolone or cortisone acetate) and was, therefore, a more homogenous group of patients for comparison.

\section{Conclusion}

In patients with adrenal insufficiency, with adverse cardiovascular profiles and impaired quality of life, replacement therapy with DR-HC, which more closely resembles the normal endogenous circadian cortisol rhythm than immediate-release $\mathrm{HC}$, offers measurable benefits with a reduction in blood pressure, weight and BMI compared with conventional HC treatment. Patients with SAI demonstrated a greater reduction in blood pressure 
compared to patients with PAI post-DR-HC treatment, which has important implications, as this cohort have excess cardiovascular mortality. Additionally, DR-HC confers significant improvements in QoL compared to immediate-release $\mathrm{HC}$, particularly in patients with primary adrenal insufficiency, which was also reflected in the patient preference for DR-HC in this study. This study highlights the potential difference in the benefits of DR-HC between patients with primary and secondary adrenal insufficiency and the need for a personalized approach to the management of patients with adrenal insufficiency receiving hydrocortisone therapy (50).

\section{Supplementary materials}

This is linked to the online version of the paper at https://doi.org/10.1530/ EJE-20-0642.

\section{Declaration of interest}

R D is funded by the Irish Research Council, The Meath Foundation and The Irish Endocrine Society. M S has received funding for this InvestigatorInitiated Study from Shire (IE). The other authors have nothing to disclose.

\section{Funding}

The Irish Research Council, The Meath Foundation, Irish Endocrine Society, Shire Ltd. The funders of the study had no role in the study design, data collection, data analysis, data interpretation, writing of the report, or the decision to submit for publication. The corresponding author had full access to all the data in the study and had the final responsibility for the decision to submit for publication.

\section{Acknowledgements}

The authors would like to thank all the study participants who kindly volunteered to participate in this study. The authors would also like to thank the staff of the RCSI Clinical Research Facility, Dublin, Ireland, and Dr Iona Galloway, Dr Maria Tomkins and Dr Liam O Murchadha, Department of Endocrinology, Beaumont Hospital, Dr Conor Woods, Department of Endocrinology, Naas General Hospital, Ireland and Professor Jeremy Tomlinson, Oxford Centre for Diabetes, Endocrinology and Metabolism, University of Oxford, UK, for their assistance during the study.

\section{References}

1 Arlt W \& Allolio B. Adrenal insufficiency. Lancet $2003 \mathbf{3 6 1}$ 1881-1893. (https://doi.org/10.1016/S0140-6736(03)13492-7)

2 Bensing S, Brandt L, Tabaroj F, Sjoberg O, Nilsson B, Ekbom A, Blomqvist P \& Kampe O. Increased death risk and altered cancer incidence pattern in patients with isolated or combined autoimmune primary adrenocortical insufficiency. Clinical Endocrinology 200869 697-704. (https://doi.org/10.1111/j.1365-2265.2008.03340.x)

3 Erichsen MM, Lovas K, Fougner KJ, Svartberg J, Hauge ER, Bollerslev J, Berg JP, Mella B \& Husebye ES. Normal overall mortality rate in Addison's disease, but young patients are at risk of premature death. European Journal of Endocrinology 2009160 233-237. (https:// doi.org/10.1530/EJE-08-0550)

4 Bergthorsdottir R, Leonsson-Zachrisson M, Oden A \& Johannsson G. Premature mortality in patients with Addison's disease: a population- based study. Journal of Clinical Endocrinology and Metabolism 200691 4849-4853. (https://doi.org/10.1210/jc.2006-0076)

5 Skov J, Sundstrom A, Ludvigsson JF, Kampe O \& Bensing S. Sexspecific risk of cardiovascular disease in autoimmune Addison disease-a population-based cohort study. Journal of Clinical Endocrinology and Metabolism 2019104 2031-2040. (https://doi. org/10.1210/jc.2018-02298)

6 Aulinas A \& Webb SM. Health-related quality of life in primary and secondary adrenal insufficiency. Expert Review of Pharmacoeconomics and Outcomes Research 201414 873-888. (https://doi.org/10.1586/14 737167.2014.963559)

7 Filipsson H, Monson JP, Koltowska-Haggstrom M, Mattsson A \& Johannsson G. The impact of glucocorticoid replacement regimens on metabolic outcome and comorbidity in hypopituitary patients. Journal of Clinical Endocrinology and Metabolism 200691 3954-3961. (https://doi.org/10.1210/jc.2006-0524)

8 Johannsson G, Nilsson AG, Bergthorsdottir R, Burman P, Dahlqvist P, Ekman B, Engstrom BE, Olsson T, Ragnarsson O, Ryberg M et al. Improved cortisol exposure-time profile and outcome in patients with adrenal insufficiency: a prospective randomized trial of a novel hydrocortisone dual-release formulation. Journal of Clinical Endocrinology and Metabolism 201297 473-481. (https://doi. org/10.1210/jc.2011-1926)

9 Johannsson G, Skrtic S, Lennernas H, Quinkler M \& Stewart PM. Improving outcomes in patients with adrenal insufficiency: a review of current and future treatments. Current Medical Research and Opinion 201430 1833-1847. (https://doi.org/10.1185/0300799 5.2014 .925865

10 Isidori AM, Venneri MA, Graziadio C, Simeoli C, Fiore D, Hasenmajer V, Sbardella E, Gianfrilli D, Pozza C, Pasqualetti P et al. Effect of once-daily, modified-release hydrocortisone versus standard glucocorticoid therapy on metabolism and innate immunity in patients with adrenal insufficiency (DREAM): a single-blind, randomised controlled trial. Lancet: Diabetes and Endocrinology 20186 173-185. (https://doi.org/10.1016/S2213-8587(17)30398-4)

11 Isidori AM, Hasenmajer V \& Venneri MA. Once-daily, modifiedrelease hydrocortisone in patients with adrenal insufficiency authors' reply. Lancet: Diabetes and Endocrinology 20186 270-271. (https://doi.org/10.1016/S2213-8587(18)30040-8)

12 Khoo B. Once-daily, modified-release hydrocortisone in patients with adrenal insufficiency. Lancet: Diabetes and Endocrinology 20186269. (https://doi.org/10.1016/S2213-8587(18)30044-5)

13 Behan LA, Rogers B, Hannon MJ, O'Kelly P, Tormey W, Smith D, Thompson CJ \& Agha A. Optimizing glucocorticoid replacement therapy in severely adrenocorticotropin-deficient hypopituitary male patients. Clinical Endocrinology 201175 505-513. (https://doi. org/10.1111/j.1365-2265.2011.04074.x)

14 Quinkler M, Miodini Nilsen R, Zopf K, Ventz M \& Oksnes M. Modifiedrelease hydrocortisone decreases BMI and $\mathrm{HbA1c}$ in patients with primary and secondary adrenal insufficiency. European Journal of Endocrinology 2015172 619-626. (https://doi.org/10.1530/EJE-14-1114)

15 Mongioi LM, Condorelli RA, La Vignera S \& Calogero AE. Dualrelease hydrocortisone treatment: glycometabolic profile and healthrelated quality of life. Endocrine Connections 20187 211-219. (https:// doi.org/10.1530/EC-17-0368)

16 Guarnotta V, Di Stefano C, Santoro A, Ciresi A, Coppola A \& Giordano C. Dual-release hydrocortisone vs conventional glucocorticoids in adrenal insufficiency. Endocrine Connections 20198 853-862. (https://doi.org/10.1530/EC-19-0176)

17 Guarnotta V, Mineo MI, Radellini S, Pizzolanti G \& Giordano C. Dual-release hydrocortisone improves hepatic steatosis in patients with secondary adrenal insufficiency: a real-life study. Therapeutic Advances in Endocrinology and Metabolism 201910 2042018819871169. (https://doi.org/10.1177/2042018819871169)

18 Ceccato F, Selmin E, Sabbadin C, Dalla Costa M, Antonelli G, Plebani M, Barbot M, Betterle C, Boscaro M \& Scaroni C. Improved 
salivary cortisol rhythm with dual-release hydrocortisone. Endocrine Connections 20187 965-974. (https://doi.org/10.1530/EC-18-0257)

19 Frara S, Chiloiro S, Porcelli T, Giampietro A, Mazziotti G, De Marinis L \& Giustina A. Bone safety of dual-release hydrocortisone in patients with hypopituitarism. Endocrine 201860 528-531. (https:// doi.org/10.1007/s12020-017-1512-1)

20 Boffa RJ, Constanti M, Floyd CN, Wierzbicki AS \& Guideline Committee. Hypertension in adults: summary of updated NICE guidance. BMJ 2019367 15310. (https://doi.org/10.1136/bmj.15310)

21 Brazier JE, Harper R, Jones NM, O'Cathain A, Thomas KJ, Usherwood T \& Westlake L. Validating the SF-36 health survey questionnaire: new outcome measure for primary care. BMJ 1992305 160-164. (https://doi.org/10.1136/bmj.305.6846.160)

22 Oksnes M, Bensing S, Hulting AL, Kampe O, Hackemann A, Meyer G, Badenhoop K, Betterle C, Parolo A, Giordano R et al. Quality of life in European patients with Addison's disease: validity of the disease-specific questionnaire AddiQoL. Journal of Clinical Endocrinology and Metabolism 201297 568-576. (https://doi. org/10.1210/jc.2011-1901)

23 Hunt SM, McKenna SP, McEwen J, Backett EM, Williams J \& Papp E. A quantitative approach to perceived health status: a validation study. Journal of Epidemiology and Community Health 198034 281-286. (https://doi.org/10.1136/jech.34.4.281)

24 Bornstein SR, Allolio B, Arlt W, Barthel A, Don-Wauchope A, Hammer GD, Husebye ES, Merke DP, Murad MH, Stratakis CA et al. Diagnosis and treatment of primary adrenal insufficiency: an Endocrine Society clinical practice guideline. Journal of Clinical Endocrinology and Metabolism 2016101 364-389. (https://doi. org/10.1210/jc.2015-1710)

25 Schofl C, Mayr B, Maison N, Beuschlein F, Meyer G, Badenhoop K, Kienitz T \& Quinkler M. Daily adjustment of glucocorticoids by patients with adrenal insufficiency. Clinical Endocrinology 201991 256-262. (https://doi.org/10.1111/cen.14004)

26 Johannsson G, Lennernas H, Marelli C, Rockich K \& Skrtic S. Achieving a physiological cortisol profile with once-daily dualrelease hydrocortisone: a pharmacokinetic study. European Journal of Endocrinology 2016175 85-93. (https://doi.org/10.1530/ EJE-15-1212)

27 Johannsson G, Bergthorsdottir R, Nilsson AG, Lennernas H, Hedner T $\&$ Skrtic S. Improving glucocorticoid replacement therapy using a novel modified-release hydrocortisone tablet: a pharmacokinetic study. European Journal of Endocrinology 2009161 119-130. (https:// doi.org/10.1530/EJE-09-0170)

28 Dunne FP, Elliot P, Gammage MD, Stallard T, Ryan T, Sheppard MC \& Stewart PM. Cardiovascular function and glucocorticoid replacement in patients with hypopituitarism. Clinical Endocrinology $1995 \mathbf{4 3}$ 623-629. (https://doi.org/10.1111/j.1365-2265.1995.tb02928.x)

29 Danilowicz K, Bruno OD, Manavela M, Gomez RM \& Barkan A. Correction of cortisol overreplacement ameliorates morbidities in patients with hypopituitarism: a pilot study. Pituitary $2008 \mathbf{1 1}$ 279-285. (https://doi.org/10.1007/s11102-008-0126-2)

30 Peacey SR, Guo CY, Robinson AM, Price A, Giles MA, Eastell R \& Weetman AP. Glucocorticoid replacement therapy: are patients over treated and does it matter? Clinical Endocrinology 199746 255-261. (https://doi.org/10.1046/j.1365-2265.1997.780907.x)

31 Sherlock M, Ayuk J, Tomlinson JW, Toogood AA, Aragon-Alonso A, Sheppard MC, Bates AS \& Stewart PM. Mortality in patients with pituitary disease. Endocrine Reviews 201031 301-342. (https://doi. org/10.1210/er.2009-0033)

32 Bates AS, Van't Hoff W, Jones PJ \& Clayton RN. The effect of hypopituitarism on life expectancy. Journal of Clinical Endocrinology and Metabolism 199681 1169-1172. (https://doi.org/10.1210/ jcem.81.3.8772595)

33 Bulow B, Hagmar L, Mikoczy Z, Nordstrom CH \& Erfurth EM Increased cerebrovascular mortality in patients with hypopituitarism. Clinical Endocrinology 199746 75-81. (https://doi. org/10.1046/j.1365-2265.1997.d01-1749.x)

34 Rosen T \& Bengtsson BA. Premature mortality due to cardiovascular disease in hypopituitarism. Lancet 1990336 285-288. (https://doi. org/10.1016/0140-6736(90)91812-o)

35 Deepak D, Furlong NJ, Wilding JP \& MacFarlane IA. Cardiovascular disease, hypertension, dyslipidaemia and obesity in patients with hypothalamic-pituitary disease. Postgraduate Medical Journal 200783 277-280. (https://doi.org/10.1136/pgmj.2006.052241)

36 Ettehad D, Emdin CA, Kiran A, Anderson SG, Callender T, Emberson J, Chalmers J, Rodgers A \& Rahimi K. Blood pressure lowering for prevention of cardiovascular disease and death: a systematic review and meta-analysis. Lancet 2016387 957-967. (https://doi.org/10.1016/S0140-6736(15)01225-8)

37 Stewart PM \& Edwards CR. The cortisol-cortisone shuttle and hypertension. Journal of Steroid Biochemistry and Molecular Biology 199140 501-509. (https://doi.org/10.1016/0960-0760 (91)90269-b)

38 Margolis KL, Manson JE, Greenland P, Rodabough RJ, Bray PF, Safford M, Grimm RH, Jr, Howard BV, Assaf AR, Prentice R et al. Leukocyte count as a predictor of cardiovascular events and mortality in postmenopausal women: the Women's Health Initiative Observational Study. Archives of Internal Medicine 2005165 500-508. (https://doi.org/10.1001/archinte.165.5.500)

39 Zalokar JB, Richard JL \& Claude JR. Leukocyte count, smoking, and myocardial infarction. New England Journal of Medicine 1981304 465-468. (https://doi.org/10.1056/NEJM198102193040806)

40 Danesh J, Collins R, Appleby P \& Peto R. Association of fibrinogen, C-reactive protein, albumin, or leukocyte count with coronary heart disease: meta-analyses of prospective studies. JAMA 1998279 1477-1482. (https://doi.org/10.1001/jama.279.18.1477)

41 Sabatine MS, Morrow DA, Cannon CP, Murphy SA, Demopoulos LA, DiBattiste PM, McCabe CH, Braunwald E \& Gibson CM. Relationship between baseline white blood cell count and degree of coronary artery disease and mortality in patients with acute coronary syndromes: a TACTICS-TIMI 18 (Treat Angina with Aggrastat and determine Cost of Therapy with an Invasive or Conservative Strategy-Thrombolysis in Myocardial Infarction 18 trial) substudy. Journal of the American College of Cardiology 200240 1761-1768. (https://doi.org/10.1016/s0735-1097(02)02484-1)

42 Rana JS, Boekholdt SM, Ridker PM, Jukema JW, Luben R, Bingham SA, Day NE, Wareham NJ, Kastelein JJ \& Khaw KT. Differential leucocyte count and the risk of future coronary artery disease in healthy men and women: the EPIC-Norfolk Prospective Population Study. Journal of Internal Medicine 2007262 678-689. (https://doi.org/10.1111/j.1365-2796.2007.01864.x)

43 Wheeler JG, Mussolino ME, Gillum RF \& Danesh J. Associations between differential leucocyte count and incident coronary heart disease: 1764 incident cases from seven prospective studies of 30,374 individuals. European Heart Journal 200425 1287-1292. (https://doi. org/10.1016/j.ehj.2004.05.002)

44 Westerbacka J, Corner A, Tiikkainen M, Tamminen M, Vehkavaara S, Hakkinen AM, Fredriksson J \& Yki-Jarvinen H. Women and men have similar amounts of liver and intra-abdominal fat, despite more subcutaneous fat in women: implications for sex differences in markers of cardiovascular risk. Diabetologia 200447 1360-1369. (https://doi.org/10.1007/s00125-004-1460-1)

45 Kim WR, Flamm SL, Di Bisceglie AM, Bodenheimer HC \& Public Policy Committee of the American Association for the Study of Liver Disease. Serum activity of alanine aminotransferase (ALT) as an indicator of health and disease. Hepatology 200847 1363-1370. (https://doi.org/10.1002/hep.22109)

46 Giordano R, Guaraldi F, Marinazzo E, Fumarola F, Rampino A, Berardelli R, Karamouzis I, Lucchiari M, Manetta T, Mengozzi G et al. Improvement of anthropometric and metabolic parameters, and 
quality of life following treatment with dual-release hydrocortisone in patients with Addison's disease. Endocrine 201651 360-368. (https://doi.org/10.1007/s12020-015-0681-z)

47 Hahner S, Loeffler M, Bleicken B, Drechsler C, Milovanovic D, Fassnacht M, Ventz M, Quinkler M \& Allolio B. Epidemiology of adrenal crisis in chronic adrenal insufficiency: the need for new prevention strategies. European Journal of Endocrinology 2010162 597-602. (https://doi.org/10.1530/EJE-09-0884)

48 Grossman A, Johannsson G, Quinkler M \& Zelissen P. Therapy of endocrine disease: perspectives on the management of adrenal insufficiency: clinical insights from across Europe. European Journal of Endocrinology 2013169 R165-R175. (https://doi.org/10.1530/ EJE-13-0450)

49 Hahner S, Loeffler M, Fassnacht M, Weismann D, Koschker AC, Quinkler M, Decker O, Arlt W \& Allolio B. Impaired subjective health status in 256 patients with adrenal insufficiency on standard therapy based on cross-sectional analysis. Journal of Clinical Endocrinology and Metabolism 200792 3912-3922. (https://doi. org/10.1210/jc.2007-0685)

50 Stewart PM. Modified-release hydrocortisone: is it time to change clinical practice? Journal of the Endocrine Society 20193 1150-1153. (https://doi.org/10.1210/js.2019-00046)

Received 9 June 2020

Revised version received 22 October 2020

Accepted 16 November 2020 